\title{
Future of immune checkpoint inhibitors: focus on tumor immune microenvironment
}

\author{
Yunlong Jia ${ }^{1,2}$, Lihua Liu ${ }^{1,2}$, Baoen Shan ${ }^{2,3}$ \\ ${ }^{1}$ Department of Tumor Immunotherapy, ${ }^{2}$ Hebei Cancer Institute, ${ }^{3}$ Research Center, Fourth Hospital of Hebei Medical University, Shijiazhuang, China \\ Contributions: (I) Conception and design: L Liu, B Shan; (II) Administrative support: L Liu, B Shan; (III) Provision of study materials or patients: Y \\ Jia, L Liu; (IV) Collection and assembly of data: Y Jia; (V) Data analysis and interpretation: Y Jia; (VI) Manuscript writing: All authors; (VII) Final \\ approval of manuscript: All authors. \\ Correspondence to: Lihua Liu; Baoen Shan. Fourth Hospital of Hebei Medical University, Shijiazhuang, China. Email: cdlihualiu@aliyun.com; \\ baoenshan62@sina.com.
}

\begin{abstract}
Immunotherapy has become a powerful clinical strategy in cancer treatment. Immune checkpoint inhibitors (ICIs) have opened a new era for cancer immunotherapy. Nowadays, the number of immunotherapy drug approvals has increased, with numerous treatment options in clinical and preclinical development. However, there remain some obstacles to improve the efficacy of ICIs further. The tumor immune microenvironment (TIME) consists of cancer cell, immune cells and cytokines, et cetera. The dynamics of TIME determine the efficacies of ICIs. Although the ICIs showed manageable toxicity, immunerelated adverse effects (irAEs) are still unignorable for clinicians. Since some primary resistance mechanisms exist in TIME, ICIs can only show effects in individual cancer patients. Even for the patients who responded, acquired resistance will occur to neutralize the effect of ICIs. Understanding how to increase the response rates and overcome the resistance to various classes of ICIs is the key to improving clinical efficacy. Besides the novel ICIs in development, there are some approaches to establish combination therapies are underway to improve further the efficacies of ICIs in treating cancer patients. Here, we describe the complicated TIME and state quo of ICIs to prospect the future of ICIs in cancer treatment.
\end{abstract}

Keywords: Immune checkpoint inhibitor (ICI); tumor immune microenvironment (TIME); immune-related adverse effect (irAE); combination therapy

Submitted Apr 01, 2020. Accepted for publication Jul 04, 2020.

doi: 10.21037/atm-20-3735

View this article at: http://dx.doi.org/10.21037/atm-20-3735

\section{Introduction}

Cancer has been the second leading cause of death worldwide (1). Cancers arise from malignantly transformed normal cells. Sustaining proliferative signaling, evading growth suppressors, enabling replicative immortality, et cetera. are essential hallmarks of cancer, among which, immune escape is a particularly critical phase of carcinogenesis (2). The cells undergo rigorous surveillance of the immune system. When normal cells are transformed into malignant cells, they can be eradicated efficiently (2). However, these associated mechanisms protecting against the development of malignancy can promote the selection of tumor cells, simultaneously. The weakly immunogenic malignant cells escaping from immune surveillance and elimination are in hibernation to strike a balance with the immune system. These cells can gradually break the balance between the transformed cells and immunity via sculpting the tumor immune microenvironment (TIME) (3). Consequently, the immune system loses the potential to recognize and eradicate these kinds of malignant cells and let them form clinically visible cancers.

Thus, cancer immunotherapy, which was developed based on studies of the mechanisms of tumor escape, can manipulate the immune system to remodel the balance between malignant cells and immunity. The early efforts 
to manipulate the immune system to fight against cancer were pioneered by Dr. William B. Coley in the 1890s (4). However, the lack of knowledge of tumor biology limited the advancements of cancer immunotherapy. The orientation of cancer treatments turned to be more effective, while more direct methods such as radiotherapy and chemotherapy were used. Fortunately, investigations persisted in elucidating the interactions between immunity and cancer. The recent breakthroughs in the comprehension of the tumor immunology and molecular biology accelerated the development of cancer immunotherapy strategies. Some subsequent approaches to cancer immunotherapy targeted cytokines to stimulate the functions of immune cells. For instance, interleukin-2 (IL-2) is regarded as the most promising cytokines to treat cancers since it can activate and promote the growth of $\mathrm{T}$ lymphocytes and natural killer (NK) cells (5). Due to its remarkable therapeutic effects, recombinant IL-2 was approved by the US Food and Drug Administration (FDA) for metastatic renal cancer and metastatic melanoma as an effective immunotherapy agent (6). Nevertheless, owing to the high rate of failure for vaccine clinical trials, progress in the field of cancer immunotherapy stalled at the beginning of the 21 st century (7).

The identification of immune checkpoint programmed death-1 (PD-1) and cytotoxic T lymphocyte antigen-4 (CTLA4) opened a new era of cancer immunotherapy (8). PD-1 and CTLA4 effectively block the activation of T cells. Thus, the strategies neutralizing these two pathways can release the $\mathrm{T}$ cells from suppression and restore their anti-tumor immunity $(9,10)$. Over the past several years, many immune checkpoint inhibitors (ICIs) against CTLA4 and PD-1 and its ligand PD-L1 have been developed and applied to clinical practice, such as Ipilimumab, Pembrolizumab, Nivolumab, Atezolizumab, et cetera (8). Even though ICIs have improved some cancer patients' prognosis significantly, their overall clinical application status still is unsatisfactory. The obstacles in improving the efficacy of ICIs include low response rate, primary or acquired resistance, immune-related adverse event (irAE), et cetera (11).

TIME, which is a dynamic setting that is consistent with changing, plays a crucial role in the efficacy and adverse effect of ICIs (12). The main elements of TIME include cancer cells, antigen, immune cells, cytokines, et cetera, these components interact with each other to determine the tendency of anti-tumor immunity (13). Although the components of TIME are not identical among distinct kinds of cancers, the dynamics and functions of TIME present absolute consistency (14).

In this review, we provide a brief overview of TIME. We then focus on the state quo of ICIs and then discuss the challenges faced in the clinical translation of ICIs from the TIME aspect. Our overarching aim throughout this review is to provide insights into how to optimize the application of ICIs based on the concept of modulating TIME to improve the prognosis of cancer patients ultimately.

\section{Tumor immune microenvironment}

\section{T cells}

T cells are dominant participants of anti-tumor immunity, hence the $\mathrm{T}$ cell-mediated immunity determines the ultimate vigor of tumor-immunity balance. The $\mathrm{T}$ cell activation signal system contains two essential signals. The first signal is transduced by $\mathrm{T}$ cell receptor (TCR) after recognizing antigen along with the major histocompatibility complex (MHC) presented on the surface of antigenpresenting cells (APCs) (15). Simultaneously, the engagement of CD28 on the T cell surface with B7 family molecules (CD80 and CD86) on the APCs supplies the costimulatory signals as the second signal (16). After initiation by these two signals, the activated $\mathrm{T}$ cells begin to attack and destroy the cancer cells which express tumor-specific antigens. Simultaneously, some immune-suppressive mechanisms also exist to break the immunity for preventing it is overactivated (13). However, these immune-suppressive mechanisms can be hijacked by cancers to compromise the immune response toward malignancy via blocking proliferation and inducing the energy of CTLs, which eventually help to form an immunosuppressive TIME (2). The immune-suppressive factors include immune cellderived and tumor cell-derived.

PD-1 is one of the most well-studied immune checkpoints expressed on activated T cells. PD-1, belonging to the CD28 family, has two ligands, PD-L1 (also known as B7-H1) and PD-L2, with different expression patterns (17). There are still some controversies about the functions of PD-L2 in tumor immunity. Thus, the current studies focus on PD-L1, dominantly. The binding of PD-1 and PD-L1 releases an immune-inhibitory signal suppressing activation and proliferation of $\mathrm{T}$ cells (18). $\mathrm{T}$ cell activation could be suppressed by B7-H4 (also known as B7-S1), a relatively new member of the B7 superfamily that shares approximately $25 \%$ amino acid homology 
with other B7 family members in the extracellular portion (19). Although the receptor for B7-H4 remains unknown, B7-H4 expressing cells and its-immunoglobulin fusion proteins could inhibit proliferation and cytokine production of $\mathrm{T}$ cells from suppressing $\mathrm{T}$ cell-mediated anti-tumor immunity (20). Also, a V-domain Ig- containing suppressor of $\mathrm{T}$ cell activation (VISTA, also known as $\mathrm{PD}-1 \mathrm{H})$ is another recently-discovered immune checkpoint that shares molecular similarities with PD-1 (21). VISTA can be found on activated $T$ cells, neutrophils, and macrophages. Emerging studies have revealed that VISTA can function as both a ligand on APCs and a receptor on $\mathrm{T}$ cells in regulating immune responses $(22,23)$.

Furthermore, lymphocyte activation gene 3 (LAG3) is another immune checkpoint, which can be found on activated T cells, Tregs, B cells, NK cells, and plasmacytoid dendritic cells (pDC) (24). The structure of LAG3 is like CD4 protein. Consequently, it can competitively bind to the antigen-MHC class II complex on APCs with higher affinity. Thus, the T cells cannot be efficiently activated, consequently (25). B Besides the above factors, the immunoglobulin and mucin domain (TIM) family are another group of immune-suppressing proteins, among which TIM-3 was shown to be the most potent immunosuppressive activity in cancers (26).

Besides the effector $\mathrm{T}$ cells, the thymus can also derive regulatory $\mathrm{T}$ cells (Tregs) from expressing the IL-2 receptor $\alpha$-chain (also known as CD25) and the transcription factor forkhead box p3 (FOXP3). Tregs can suppress $\mathrm{T}$ cell responses through the secretion of some inhibitory cytokines such as IL-10, IL-35, and transforming growth factor- $\beta$ (TGF- $\beta$ ) or by direct cell contact (27). PD-1 expression can promote naïve CD4+ $\mathrm{T}$ cells to convert to Tregs by inhibiting the mTOR-Akt signaling cascade (28). The majority of Tregs express CTLA4. These Treg can bind to APCs and decrease their costimulatory molecules (e.g., CD80 and CD86) and, therefore, deprive stimulatory signal to $\mathrm{T}$ cells (29).

Although PD-L1 is mainly expressed on immune cells such as activated $\mathrm{T}$ cells and macrophages, many types of cancer cells express PD-L1 to generate an immunosuppressive TIME to avoid the destiny of being eliminated by cytotoxic T lymphocytes (CTLs) (18). The expression of PD-L1 on cancer cells is regulated by multiple mechanisms involving numerous regulatory levels, such as intrinsic control and extrinsic control. The former includes genomic alterations, epigenetic regulation, and oncogenic signaling activation, while the latter mainly refers to cytokines (30). Genomic alterations induce PD-L1 expression in cancer, which includes copy number alterations (CNAs) and gene polymorphism, which are both commonly observed in cancers $(31,32)$. Budczies et al. showed that gains of copy numbers of CD274, which encoding PD-L1, occurred in breast, bladder, colorectal, cervical, and ovarian carcinomas. In contrast, the deletion of CD274 occurred in melanoma and non-small cell lung cancer (NSCLC) patients (31). The PD-L1 expressionrelated epigenetic regulatory mechanisms include DNA methylation, histone modification, and non-coding RNAs. DNA methylation of the $P D-L 1$ promoter can often be observed in melanoma, head, and neck squamous cell carcinoma, and lung cancer (33-35). The histone modifications involved in CD274 gene regulation include histone deacetylases (HDACs) and histone methylation (H3K4me3), which both down-regulated the PD-L1 expression in cancer cells $(36,37)$. Also, non-coding RNAs are important parts of epigenetic mechanisms, including microRNAs (miRNAs), long non-coding RNAs (lncRNAs), and circular RNAs (cirRNAs). miRNAs can trigger target mRNA degradation or translation inhibition by targeting the 3'-untranslated regions (UTRs) (38). miR-148a-3p, miR-146a-3p, miR-873-5p were reported to downregulate PD-L1 in colorectal cancer, melanoma, breast cancer, respectively (39-41). Some lncRNAs can act as competing endogenous RNAs (ceRNAs) sponging miRNAs to form a regulatory axis for indirectly regulating the expression of PD-L1 in cancers, for instance, MIR17HG/miR-17$5 \mathrm{p}$ axis in colorectal cancer, the linc00473/miR-195-5p axis in pancreatic cancer, MALAT1/miR-200a-3p axis in NSCLC $(42,43)$. Furthermore, the oncogenic signaling activation plays pivotal roles in PD-L1 expression in some cancers. These oncogenic transcription factors include AP-1, MYC, IRF1 (interferon regulatory factor 1) and NF- $\kappa B$, etc. (44). In NSCLC, the activation of mutated EGFR can upregulate the PD-L1 upregulation on cancer cells, and this phenomenon contributes to EGFR-TKI resistance (45). In this regulatory axis, the presence of tumor suppressor bridging integrator-1 (BIN1) can partially block the downstream signal of mutated EGFR and subsequently inhibited the upregulation of PD-L1 on NSCLC cells (46). As for the cytokines, interferon- $\gamma$ (IFN- $\gamma$ ), IFN- $\alpha$, IFN- $\beta$, tumor necrosis factor- $\alpha$ (TNF- $\alpha$ ), TGF- $\beta$, IL-4, IL-17, and IL-27 could induce upregulation of PD-L1, among which IFN- $\gamma$ shows the most potent potential (47). IFN- $\gamma$ can activate the JAK/STAT and NF- $\mathrm{KB}$ pathway to upregulate PD-L1 expression on cancer cells (48). Since these 
cytokines can affect and reflect the dynamics of TIME, they often determine the expression status of PD-L1 during cancer progression and treatment.

\section{NK cells}

NK cells are founding members of the innate lymphoid cell (ILC) family (49). NK cells have been confirmed to play critical roles in anti-tumor immunity. Since antigen recognition is not essential for NK cells to exert functions, they can eliminate the abnormal and malignant cells without prior sensitization, and even preferentially kill cancer stem cells $(50,51)$. NK cells can form immune synapses with target cancer cells, thereby to release preformed cytolytic granules (e.g., perforin and granzymes) to induce cell lysis (52). However, numerous factors in TIME limit the function of NK cells. First, the function of NK cells can be impaired by Tregs. For instance, Tregs can induce apoptosis of NK cells by secreting $\beta$-galactoside-binding protein $(\beta \mathrm{GBP})$ to escape from immunity and increase lung metastasis of breast cancer cells (53). Furthermore, the T cell-suppressing factors, including PD-1, CTLA4, TIM-3, and LAG3, can bind to their ligand and transmit inhibitory signals to dampen NK cell activation (52,54). Last but the most important, NK cells can be disabled by specific HLA class I-specific inhibitory receptors, such as killer-cell immunoglobulin-like receptors (KIRs) and NKG2 family.

NKG2A is an intracytoplasmic tyrosine-based inhibitory motif (ITIM)-bearing receptor, which is phosphorylated and recruits the phosphatases responsible for transmitting the inhibition signal to immune effector cells. In healthy individuals, only $5 \%$ of $\mathrm{CD}^{+} \mathrm{T}$ lymphocytes in peripheral blood express NKG2A at a steady state, but this expression can be upregulated by chronic antigenic stimulation (55). Commonly, NKG2A is expressed at the cell surface and form a heterodimer with CD94 and recognizes the nonclassical class I major histocompatibility complex (MHC-I) molecules (56). Binding of NKG2A/CD94 to its cognate ligand can inhibit the effect of $\mathrm{T}$ lymphocytes and NK cells via an SHP-1-dependent inhibition signal transmission (57). The ligand of NKG2A is HLA-E in humans and Qa-1 in the mouse. Some factors inhibiting the function of $\mathrm{T}$ cells can also suppress the NK cells, such as LAG. Approximately half of NK cells in peripheral blood express NKG2A.

\section{Myeloid cells}

Myeloid-derived suppressor cells (MDSCs) are reported to be a significant regulator of the immune response in various diseases, including cancer (58). These cells derive from hematopoietic stem cells (HSCs) existing in bone marrow, which induce an immature myeloid cell population (59). It has been shown that hypoxia-induced factor $1 \alpha(\mathrm{HIF} 1 \alpha)$ is upregulated in MDSCs that are essential for their immunosuppressive function in TIME (60). In TIME, the recruitment and activation of MDSCs depend on vascular endothelial growth factor (VEGF), G-CSF, GM-CSF, TGF- $\beta$, IL-10, etc. (61). The expression of VISTA increased 10-fold on MDSCs in TIME, compared to those in the peripheral lymph node (62).

Also, MDSCs are the principal cells to derive tumorassociated macrophages (TAMs). Besides MDSCs, mononuclear cells and tissue-resident macrophages can also derive TAMs. TAMs are the most abundant population of tumor-infiltrating immune cells in TIME (63). According to the polarization status, macrophages can be divided into two subtypes: the M1 subtype promoting anti-tumor immunity, and the M2 subtype exerting pro-tumorigenic effects (64). In detail, M1 macrophages play critical roles in killing tumor cells by producing reactive oxygen/nitrogen species (ROS/RNS) and pro-inflammatory cytokines such as TNF- $\alpha$, IL-1 $\beta$, and IL-6 (65). M1 and M2 macrophages can be distinguished based on the differential expression of transcription factors. Furthermore, they are also distinguished by surface molecules and the disparities in their cytokine profile and metabolism (66). M1 macrophages polarization are mainly mediated by Th1 cytokines and activated Toll-like receptors (TLRs), and M2 macrophage polarization, which are induced by Th2 cytokines such as IL-4, IL-10, and IL-13 $(65,67)$. Although the antitumor effect is the dominant function of M1 macrophages, numerous studies emerge to prove that they also exert protumor effects. M1 macrophages are reported to induce PDL1 expression in hepatocellular carcinoma and promote immune escape (HCC) (68). M2 macrophages are divided into four phenotypes, including M2a, M2b, M2c, and M2like, among which M2-like plays the most crucial role in cancers. M2-like macrophages can be activated by growth factors and cytokines to turn into M2d subtype with the immunosuppressive role and pro-tumor function in TIME (67). During this process, the activation of colonystimulating factor 1 receptor (CSF1R) signaling in TAMs may play crucial roles (69).

In general, TAMs tend to present the characteristics of M2 macrophages with Th2 immune response (70). Clinical studies have confirmed that higher frequencies 
of TAMs are associated with poor prognosis of cancer patients (70). TAMs took part in promoting cancer initiation and development via numerous pathways, such as pro-angiogenesis, stimulating epithelial-mesenchymal transition (EMT), and immunosuppression (71). TAMs can suppress naïve $T$ cell proliferation in vitro and inhibit cytotoxic $\mathrm{T}$ cell response directly. TAMs inhibit $\mathrm{T}$ cell function by expressing Arginase 1 (ARG1) to exhaust $\mathrm{L}$-arginine, which is essential for the activation of $\mathrm{T}$ cells. L-arginine depletion leads to the failure to re-express CD3 $\zeta$-chain in the TCR complex, thus suppressing effector $\mathrm{T}$ cell activation (72). In TIME, TAMs are influenced by some tumor-derived factors to secrete an array of immune-suppressive cytokines, such as IL-10, TGF- $\beta$, and prostaglandin-E2 (PGE2), which modulate the TIME to inhibit $\mathrm{T}$ cell-mediated immune responses (73). Besides secreting immune-suppressing factors, TAM also induces immune suppression by expressing inhibitory receptors, including classical and non-classical MHC-I molecules, which are regarded to be associated with the antigen presentation process. Macrophages can express human leukocyte antigen (HLA) molecules, such as HLA-C, HLA-E, and HLA-G, which could bind to CD94 and leukocyte immunoglobulin-like receptor subfamily B member1 (LIR1) to inhibit the activation of NK cells and a subset of activated T cells, respectively (63). Simultaneously, TAMs also express immune checkpoints, including PDL1, PD-L2, CD80, CD86, and B7-H4 $(63,66)$. Otherwise, TAMs can inhibit the function of effector $T$ cells by recruiting natural Tregs to the TIME and sustain their survival through the secretion of $\mathrm{C}-\mathrm{C}$ chemokine ligands (CCLs), including CCL-2, CCL-3, CCL-4, CCL5, CCL20 and CCL-22 (74).

Besides, emerging evidence shows that tumor-associated neutrophils (TANs) play crucial roles in regulating antitumor immunity. TANs exert different functions according to their polarization states. Briefly, the N1 phenotype is cytotoxic toward tumor cells by producing a high level of TNF- $\alpha, \mathrm{H}_{2} \mathrm{O}_{2}$, and Fas, which mainly exist in early cancers (75). When N1 neutrophils have physical contact with cancer cells, they can trigger a TRPM2 $\mathrm{Ca}^{2+}$ channeldependent cell death via secreting $\mathrm{H}_{2} \mathrm{O}_{2}$ (76). Meanwhile, the Fas expressed on N1 neutrophils can bind to the Fas ligand on tumor cells to result in a "self-destruction" mechanism (77).

However, under the factors released during the process of the cancer establishment, TANs are resculpted to be the more tumorigenic N2 phenotype (78). In TIME, the main factors inducing N2 polarization are G-CSF and TGF- $\beta(75,79)$. TANs of the N2 phenotype express a high level of arginase and derive immune-suppressing factors such as CCL2, CCL3, IL-17, and CXCL8 (80). Tsuda et al. confirmed that CCL2 and CCL3 affected the immune effector cells in TIME to form a host immune suppression setting (81). The release of CXCL8 by N2 neutrophils may suggest a feedforward loop of neutrophil recruitment (82).

The agents taken above targeting TAMs or TANs might be an ideal strategy to be combined with ICIs for improving the patient outcome.

\section{Cancer and stroma cells}

Under the selective pressure of the anti-tumor immunity, cancer cells arise to present some immune-suppressive features and sculpt the TIME to create suitable conditions. The metabolism status in TIME may determine the functions of immune cells. Indoleamine 2,3-dioxygenase (IDO) can alter the amino acid metabolism by catalyzing the tryptophan (Trp) into kynurenine (Kyn) which is detrimental to T cells (83). The upregulation of IDO in cancer cells has been regarded to be a crucial factor promoting carcinogenesis and is related with poor prognosis of cancer patients (84). Simultaneously, cancer cells can hijack the innate chemokine networks to promote immune suppression and metastatic cancer cell trafficking. Furthermore, cancer cells and stroma cells in TIME can secret exceptionally high level of VEGF, which leads to formation of abnormal vessels and impaired perfusion. VEGF can reduce interaction between immune cells and endothelial cells. These phenomena can restrict immune cells to infiltrate into TIME (85). VEGF also directly inhibits DC maturation and activation of Tregs to prevent the activation of $T$ cells (86).

Carcinoma-associated fibroblasts (CAFs) are the most prominent components of tumor stroma and mainly act as immunosuppressive cells. CAFs release some cytokines (e.g., IL-6 and TGF- $\beta$ ) and chemokines (e.g., CXCL1, CXCL12 and CCL2) which are necessary to attract and retain suppressive immune cells into the TIME (87). Meanwhile, CAFs can attract macrophages, T cells and NK cells toward the juxtatumoral stroma and trapped them to prevent the immunological access to TIME (88). Besides direct effects, CAFs also exhibit immune suppressive functions via inducing polarization of resident macrophages and neutrophils in TIME toward M2 and N2 subtype (89). Thus, targeting CAFs might benefit the ICIs in clinical 
conditions by relieving immune suppression and promoting immune cell infiltration.

\section{State quo of ICls}

\section{Single-agent therapy}

The ICIs approved by the FDA to treat cancers include the agents targeting PD-1, PD-L1, and CTLA4, respectively. Nivolumab (Opdivo) and Pembrolizumab (Keytruda) are anti-PD-1 agents approved by FDA in 2014, followed by anti-PD-L1 agents Atezolizumab (Tecentriq), Durvalumab (Imfinzi) and Avelumab (Bavencio) approved in 2018 (90). Ipilimumab is the first anti-CTLA4 agent for clinically commercial use (91). Since the TIME varies by cancer and the agent molecular structures are not entirely the same, the efficacies of these ICIs are also different among various cancers. Present studies have confirmed that the cancers harboring highly immunogenic mutations are sensitive to ICIs, such as melanoma, renal cell carcinoma (RCC), and NSCLC (92). For instance, in the above three cancers, the overall survival in the cohort of phase I clinical trial for Nivolumab is 9.9, 16.8, and 22.4 months, respectively (93).

In 2016, FDA permitted Pembrolizumab as a single agent for first-line treatment of metastatic NSCLC patients with $\mathrm{PD}-\mathrm{L} 1 \geq 50 \%$, according to the results from KEYNOTE-024 phase III trial (94). Also, results from KEYNOTE-042 phase III trial revealed that Pembrolizumab could even improve the metastatic NSCLC patients with PD-L1 $\geq 1 \%$ (95). However, Nivolumab exhibited no significantly better effect on improving the overall survival (OS) or progression-free survival than chemotherapy in previously untreated patients at stage IV or recurrent patients with PD-L1 $\geq 5 \%$ and even PD-L1 $\geq 50 \%$ (96). As for the second-line treatment, Nivolumab and Pembrolizumab also exhibited promising effects. Results from the CHECKMATE-017 trial showed that Nivolumab significantly improved the objective response rate (ORR), OS, and PFS in patients with previously treated advanced squamous NSCLC, compared to docetaxel (97).

Furthermore, Nivolumab also achieved a better ORR and OS than docetaxel in nonsquamous NSCLC patients according to the results from the CHECKMATE-057 trial (98). Atezolizumab can further improve the PFS and OS in patients with metastatic nonsquamous NSCLC, regardless of PD-L1 expression and EGFR or ALK genetic alteration status, according to the ATLANTIC phase II trial (99). The results from the OAK trial showed that
Atezolizumab had a clinically relevant effect on OS versus docetaxel in previously treated NSCLC patients, regardless of PD-L1 expression or histology (100).

For treating melanoma, the ICIs also exhibit promising effects. Nivolumab is the first anti-PD-1 antibody approved by the FDA in 2014 for treating melanoma according to the results from the CHECKMATE-066 (101). The results showed that Nivolumab achieved better ORR, PFS, and 1 -year survival rate in the previously untreated patients with metastatic melanoma and wild-type BRAF, compared to the dacarbazine (101). Pembrolizumab also exhibited a satisfactory effect in treating metastatic melanoma. Results from KEYNOTE-001 trial demonstrated that the ORR was $52 \%$ with $25 \%$ complete remission (CR), and the disease control rate (DCR) was $72 \%$. The median PFS was 8.3 months in all patients and 16.9 months in treatmentnaivve patients, and the estimated 5-year OS rate was 34\% and $41 \%$, respectively (102). Furthermore, Pembrolizumab can be applied as adjuvant therapy for high-risk stage III melanoma with significantly longer recurrence-free survival than placebo (103). FDA approved Ipilimumab in 2015 as an adjuvant therapy for patients with regional lymph node metastases who have undergone complete resection according to the results from EORTC 18071 phase III trial (104).

ICIs have been the central part of treating clear cell renal cell cancer (ccRCC). CHECKMATE-025 phase III trial enrolled 821 patients with advanced ccRCC patients who had received one or two prior regimens (including at least one targeting VEGFR). The results showed that Nivolumab achieved better ORR than everolimus with a significant odds ratio of 5.98. Meanwhile, the OS and PFS were longer in the Nivolumab group than the everolimus group, regardless of PD-L1 expression (105).

ICIs also achieved promising efficacies in some other cancers, including triple-negative breast cancer (TNBC), bladder cancer and head and neck squamous cell cancer (HNSCC). The TNBC cohort in KEYNOTE-012 phase Ib trial enrolled 32 cases of heavily pre-treated patients with $\mathrm{PD}-\mathrm{L} 1$ positivity in the stroma or $\mathrm{PD}-\mathrm{L} 1 \geq 1 \%$ tumor cells using their Qualtek assay. The results showed that the ORR of the patients receiving Pembrolizumab was $18.5 \%$, with the median time to response of 17.9 weeks (106). In another clinical trial, KEYNOTE-086, evaluating Pembrolizumab monotherapy in metastatic TNBC as firstline or above treatment, the cohort B consisting patients with previously untreated metastatic disease and PD-L1 CPS $\geq 1 \%$ had an ORR of $23 \%$, with $4 \%$ achieving a 
CR (107). Atezolizumab is the first ICI allowed by the FDA to treat advanced bladder cancer as second-line therapy. The patients receiving Atezolizumab had better ORR and OS than those treated with other second-line regimens (108).

\section{Combination therapy}

\section{Combination with radiotherapy}

In the past century, radiotherapy has been an indispensable part of cancer treatment. Currently, 50\% of all cancer patients receive radiotherapy alone or in combination with surgery or chemotherapy (109). Recently, preclinical evidence has revealed that radiotherapy can modulate the TIME via different mechanisms. First, radiotherapy can trigger immunogenic cell death (ICD), resulting in the release of neo-antigens and increase the cross-presentation of these antigens via APCs to directly stimulate T-cell responses in TIME (110). Furthermore, radiotherapy can upregulate cell surface molecules (e.g., MHC class I and CD95) and increase the secretion of IFN-1 or other components of the antigen processing machinery to enhance the recognition ability of $\mathrm{T}$ cells (111). Moreover, radiotherapy promotes the release of cytokines to decrease the presence of MDSCs and increase the infiltration of effector T cells in the TIME (112). Also, radiotherapy can upregulate the expression of pro-inflammatory cytokines and chemokines, as well as NK activating ligands in TIME (13).

However, the immune cells are commonly susceptible to radiation. Thus, they can be eliminated at much lower radiation doses than needed to kill tumor cells. Thereby, despite multiple mechanisms by which radiotherapy can activate anti-tumor immunity, high-dose radiation is immune suppressive in most clinical situations (13). Meanwhile, radiotherapy can upregulate the TIM-3 on $\mathrm{CD}^{+} \mathrm{T}$ cells and recruit Tregs in the TIME, which in turn suppresses the $\mathrm{T}$ cell-mediated anti-tumor immunity (113). Thus, the actual efficacy of radiotherapy plus ICI therapy still need to be determined in clinical trials.

Guided by these concepts, some trials were performed to reveal the potential clinical application value of radiotherapy plus ICI therapy. Results from KEYNOTE-001 phase I trial demonstrated that the PFS and OS of the patients previously received radiotherapy before Pembrolizumab were both significantly increased than those of the patients never received radiotherapy, and this improvement was regardless of radiation area or doses (114). Similarly, the results of a meta-analysis included 16 clinical trials revealed that the metastatic melanoma patients received combination therapy of radiotherapy plus Ipilimumab had higher ORR and longer OS than those received Ipilimumab alone (115). Further, radiotherapy can bring some potential benefits to those patients who are not sensitive to ICIs. For instance, despite Ipilimumab did not significantly improve the prognosis of the NSCLC patients, addition of radiotherapy could induce responses to Ipilimumab in part of NSCLC patients (115). In conclusion, combination of radiotherapy and ICIs showed great clinical applicant potential, but it is still urgent to elucidate the concrete efficacies with more specific clinical trials.

\section{Combination with chemotherapy}

Chemotherapy is another standard anti-tumor therapy widely in use worldwide for decades. Chemotherapy agents can enhance anti-tumor immunity through multiple pathways, such as exposing the tumor-associated antigens in cancer cells, promoting $T$ cells infiltration, downregulating Tregs $(116,117)$. Thus, to increase the response rate of ICIs, chemotherapy is an ideal partner to set up a combination treatment strategy. In the KEYNOTE-021 phase II trial, the ORR of Pembrolizumab plus chemotherapy (carboplatin and pemetrexed) group (55\%) is significantly higher than that of chemotherapy alone group (29\%), even in the patients with PD-L $1<1 \%$ (118). Based on these significant results, the combination of Pembrolizumab and chemotherapy (carboplatin plus pemetrexed) is under an accelerated approval process by the FDA as firstline treatment for patients with metastatic nonsquamous NSCLC (119).

Results from Impower 133 trial demonstrated that the extensive-stage small-cell lung (ES-SCLC) cancer patients receiving a combination of Atezolizumab and chemotherapy (carboplatin plus etoposide) as first-line treatment presented significantly longer OS and PFS, compared to those receiving chemotherapy alone (120). CASPIAN is a phase III trial to evaluate the potential of a combination strategy of Durvalumab and chemotherapy (platinum plus etoposide) as first-line treatment in ES-SCLC. The results showed that Durvalumab plus platinum-etoposide significantly improved the OS of ES-SCLC patients versus a clinically relevant control group (121).

Results from Impassion 130 trial revealed that Atezolizumab plus nanoparticle albumin-bound (nab)paclitaxel significantly prolonged the PFS of metastatic TNBC patients in both the intention-to-treat population and the PD-L1-positive subgroup (122). 


\section{Combination with other therapies}

Similar to radiotherapy and chemotherapy, some other therapies also showed great potential to be combined with ICIs due to their effect on altering the TIME. AntiVEGF therapy, especially Bevacizumab, is a nonnegligible part of cancer treatment and achieved amazing success in recent years. In view of the immune-suppressing effect of VEGF in TIME, anti-VEGF agent is ideal for establishing combination therapy based on ICIs. Bevacizumab significantly extended the PFS from a median of 6.1 months for ICI monotherapy to 11.7 months for the doublet in patients with previously untreated hepatocellular carcinoma (123). Another anti-VEGF agent, Axitinib, also showed outstanding efficacy as first-line treatment of advanced renal cell carcinoma (aRCC) in a phase III clinical trial (124). Since deficiency of PD-L1 contributes to antiPD-1/PD-L1 therapy, treatments upregulating PD-L1 expression in TIME might sensitize ICIs targeting PD-1/ PD-L1 axis (125). Also, the agents targeting epigenetic mechanisms are potential cooperators of ICIs. The addition of decitabine, which induced the inhibition of global methylation, could significantly improve the efficiency of anti-PD-1 therapy via upregulating constitutive PD-L1 expression (126). Furthermore, the combination of novel epigenetic agent JQ1 and anti-PD-1 agents significantly improved the anti-tumor immunity in mice bearing KRASdriven NSCLC (127).

\section{Challenge of ICI}

\section{Primary resistance}

Although there is a significant improvement in patient prognosis achieved with ICIs, the durable objective responses are variations among different cancer types and limited in only a minority of patients. Specific immunotherapy resistance mechanisms may exist in some patients before ICI treatment, and these individuals have primary resistance to ICIs. These mechanisms involve tumor cell-extrinsic factors and tumor cell-intrinsic factors. Tumor cell-extrinsic mechanisms resulting in ICI primary resistance involve components other than tumor cells within the TIME. These factors mainly apply to the cells or factors that suppress the immune system mentioned above.

Tumor cell-intrinsic factors that contribute to ICI primary resistance include expression or repression of specific genes in cancer cells that prevent infiltration or activation of immune cells within the TIME. First, since the absence of tumor neoantigens is necessary for $\mathrm{T}$ cells to recognize cancer cells, the poorly immunogenic tumors show unsatisfactory response to ICIs, for instance, pancreatic ductal adenocarcinoma (128). Furthermore, lacking infiltrating $\mathrm{T}$ cells is an essential reason for ICI primary resistance, the TIME with low TILs is also called "immune desertic landscape." IDO, an endoenzyme expressed in pDC, TAM, and cancer cells, can induce immune tolerance by inhibiting the proliferation and activation of $\mathrm{T}$ cells. The presence of IDO significantly decreased the TILs while increasing the Treg/CD $8^{+} \mathrm{T}$ cell proportion in TIME (83).

Meanwhile, stabilization of $\beta$-catenin resulting in constitutive Wnt signaling can induce $\mathrm{T}$ cell exclusion from cancers and therefore lead to ant-PD-1/anti-CTLA4 resistance (129). Also, since abundant IFN- $\gamma$ is essential for the efficacy of ICIs, some cancer patients lacking the genes (e.g., IFNGR1/2, JAK2, and IRF1) for a response to IFN- $\gamma$ are more resistant to ICIs (130). Also, the tumor cell-intrinsic factors include activation of oncogenic proteins (e.g., MAPK and $W n t / \beta$-catenin) and loss of tumor suppressor (e.g., PTEN). Activation of the MAPK pathway upregulated the secretion of immune-inhibitory proteins (e.g., IL-8 and VEGF) of cancer cells, which suppressed the recruitment and function of $\mathrm{T}$ cells (131). Besides, high density of VEGF can suppress the migration of immune cells to TIME, which is significantly correlated with formation of "cold tumor" microenvironment (132). Moreover, there is some epigenetic modification of the DNA in cancers leading to changes in gene expression of immune-related genes, which can impact antigen processing, presentation, and immune escape (11). In conclusion, some factors existing in TIME before treatment may contribute to primary ICI resistance.

\section{Acquired resistance}

The induction of long-lasting responses is regarded as a hallmark of cancer immunotherapy. However, because immunotherapy resculpts the tumor-immunity balance, some activated factors may in turn cause other immune-tolerant or inhibitory mechanisms, called acquired resistance. First, like PD-L1, IDO is also under the regulation of both intracellular and extracellular mechanisms. Since IFN- $\gamma$ is the most potent inducer for IDO in cancer cells, the release of IFN- $\gamma$ by ICIs can result in an upregulated expression of IDO in TIME, which can induce acquired resistance for ICIs (133). 
Moreover, emerging evidence suggests that cancer cells can develop acquired resistance through decreased expression or mutations in the tumor antigens, which are essential for $\mathrm{T}$ cells to exert antitumor immunity. Since $\mathrm{T}$ cells reactivated by ICIs primarily recognize mutational neoantigens, the genetic deletions, mutations, or epigenetic changes that lead to loss of expression of these mutational neoantigens can result in acquired resistance to ICIs (58). Meanwhile, loss of B2M, an essential part of HLA class I folding and transport to the cell surface, can be observed after anti-PD-1 therapy. Due to the vital role of HLA class, $\mathrm{I}$, in recognition ability of $\mathrm{T}$ cells, the genetic deficiency of B2M can lead to acquired resistance to ICIs (134). Also, some immune-suppressing factors can be upregulated after the first ICI treatment. In prostate cancer and melanoma patients following Ipilimumab therapy, elevated expression of VISTA on TILs and TAMs may be observed; suggesting that VISTA might be a compensatory resistance mechanism of ICIs (135). Of note, methylation of the $P D-L 1$ promoter enhanced by anti-PD-1 therapy results in acquired resistance to ICIs in NSCLC (136).

\section{irAE}

The release of T-cell function by ICIs may enhance $\mathrm{T}$ cell activity throughout the whole organism and therefore eases autoimmune reactions. Herein, ICIs can exacerbate preexisting processes and trigger autoimmunity in patients with genetic or acquired predisposing factors, or induce novel autoimmune disease in affected organs. These alterations can form clinical symptoms, the irAEs. Most commonly, the irAE profile consists of endocrine disorders, pneumonitis, dermatologic lesions, and colitis, but it also involves serious events such as cardiotoxicity, immune-related encephalitis, and peripheral nervous system disorder (137). irAEs typically occur within in early phase of ICI therapy ( $\leq 12$ weeks after initial therapy) and rarely occur after one year of therapy (138). The incidence of irAEs with single-agent ICI varies by agents, cancer type, and disease setting. irAEs can be variable in their onset, dynamics, and presentation and often require specific management. Briefly, minor irAEs occur in about $90 \%$ of patients receiving anti-CTLA 4 agents while in $70 \%$ of patients receiving PD-1/PD-L1 blockade agents (139). According to Common Terminology Criteria for Adverse Events (CTCAE) v5.0, irAEs are classified as low-grade (grades 1-2), high-grade (grades 3-4) and lethal (grade 5) (140). The frequency of high-grade and lethal irAEs are tripled in combination therapy, compared to ICI monotherapy (141).

Emerging evidence presents the irAE onset is positively correlated with the efficacy of PD-1/PD-L1 blockade. Studies performed in different cancers, including NSCLC, melanoma, RCC, UCC, and HNSCC, revealed that the patients who experience irAEs had superior PFS and OS compared to the patients who did not experience irAEs. Also, the ORR and DCR were prolonged in patients who experienced irAEs, compared to those who did not experience irAEs (142-144). And further analysis revealed that, for NSCLC patients, the different irAE types seem to reflect the therapeutic efficacy, the patients who experienced thyroiditis had statistically significantly longer OS and PFS, compared to those who did not experience thyroiditis (142).

Since some tumor-associated antigens are not specific, the reactivated $\mathrm{T}$ cells may target the tumor and healthy tissues harboring the same antigens. The study, including 73 NSCLC patients receiving anti-PD-1 agents, revealed that shared $\mathrm{T}$-cell clones between skin and cancer were present in all patients. Further experiments showed nine candidates shared antigens between skin and cancer, which showed the potential to elicit IFN-based $\mathrm{T}$ cells responses in stimulated peripheral blood mononuclear cells (145). Multiple mechanisms have been proposed to account for the development of irAEs, but the exact pathophysiology still is to be elucidated.

Glucocorticoids have been proved to be effective methods to manage irAEs, but they can partially neutralize the effect of ICIs (146). Therefore, finding other effective agents to manage the irAEs is urgent to improve the efficacy of ICIs further. A phase II clinical trial, combining highdose with or without GM-CSF in metastatic melanoma patients, revealed that GM-CSF showed lower toxicities and better survival with equal ORR (147). Based on this finding, a phase III study (NCT02339571) of Ipilimumab and Nivolumab with or without GM-CSF is performed. For the patients who experienced irAEs during first ICI retreatment, about $52 \%$ of cases experienced irAEs again in the ICI treatment (148).

\section{Future of $\mathrm{ICI}$}

\section{Novel ICIs}

Since CSF1R signaling is essential for TAMs to acquire the immunosuppressive and pro-tumorigenic M2-like phenotype, Pexidartinib (PLX3397) which blocks CSF1/ CSF1R signaling is a potential trial to improve the efficacy 
of ICIs (69). LAG3 blockade is regarded as a potential method to increase the responsiveness of CTLs. Nowadays, four agents are targeting LAG3 are in clinical trials as cancer treatment adjuvants, including MK-4280 (Merck), BMS-986016 (Bristol-Myers), IMP321 (Immutep) and LAG525 (Novartis) (149). IPH 2201 (Monalizumab), a humanized anti-NKG2A blocking mAb, increased degranulation and IFN- $\gamma$ production by $\mathrm{NKG}_{2} \mathrm{~A}^{+} \mathrm{NK}$ cell against HLA- $\mathrm{E}^{+}$target cells, thereby promoting $\mathrm{NK}$ cell effector functions. Since HLA-E was widely expressed on the surfaces of multiple cancer types, NKG2A blockade alone or, in combination with ICIs, might improve the anti-tumor efficacy of $\mathrm{NK}$ and $\mathrm{CD} 8^{+} \mathrm{T}$ cells in TIME, simultaneously (150). Emerging studies have proved that Monalizumab is a possible and ideal partner of ICIs for building a novel combination treatment strategy. The clinical trial NCT02671435, aiming at evaluating the toxicity and efficacy of Monalizumab plus Durvalumab, consists of a cohort of patients with metastatic microsatellite-sable (MSS) colorectal cancer, a population historically nonresponsive to PD-1/PD-L1 blockade. Preliminary data demonstrate that this combination of Monalizumab and Durvalumab has a manageable toxicity profile and encouraging activity in these patients. Meanwhile, for reactivating the NK cells, anti-KIR agents have also been developed, such as IPH2101 and IPH2102 (Lirilumab), which are clinical grade IgG4 mAbs interacting with KIR2D (151). Most clinical trials of these two agents are performed in patients with hematologic malignancies, and there is no available data about their effects when in combination with ICIs.

\section{Combination of multiple ICIs}

ICIs reactivate the $\mathrm{T}$ cells in TIME by inhibiting the immune checkpoint to exhibit therapeutic effects. However, $\mathrm{T}$ cells reactivated by anti-PD-1/PD-L1 or anti-CTLA4 agent alone are possibly inhibited by other immune suppressing cells or factors in TIME (152). For instance, the $\mathrm{T}$ cells which are reactivated by Pembrolizumab could be suppressed by CTLA4, and they still in a state of anergy in TIME, consequently. Thus, the combination therapy of multiple ICIs is a potential way to further improve the efficacies of ICIs. The results from the CHECKMATE-227 trial demonstrated that first-line treatment with Nivolumab plus Ipilimumab resulted in a longer duration of OS than chemotherapy in NSCLC patients, independent of the PD-L1 expression, despite Nivolumab alone exhibited no significantly better effects on OS than chemotherapy in advanced NSCLC patients (153). In the CHECKMATE-067 trial, the therapy-naïve patients with metastatic melanoma receiving Ipilimumab and Nivolumab had better ORR and 5-year survival rate with manageable irAEs, compared to Nivolumab or Ipilimumab alone (154). Results from CHECKMATE-032 demonstrated that, for treating patients with locally advanced or metastatic chemotherapy-refractory gastric, esophageal or gastroesophageal junction cancer, Ipilimumab further improved the ORR and 12-month PFS rate achieved by Nivolumab (155). Meanwhile, since the increase in Kyn/ Trp ratio has been regarded as an adaptive resistance mechanism associated with worse survival of patients treated with Nivolumab, targeting this metabolomic alteration caused by IDO expressed in TIME might benefit the ICIs (156). Guided with this concept, phase III EHO-301/ KEYNOTE-252 trial was performed, but the combination of Epacadostat (a selective IDO1 inhibitor) did not exhibit satisfactory results, unfortunately (157). A timely and appropriate monitoring serum Kyn/Trp should be highlighted to evaluate and select patients who are proper for combination of Epacadostat and anti-PD-1 agents (156). Taken above, for cancers with a certain status, the combination of various ICIs can overcome some problems of ICI monotherapy.

\section{Patient selection}

Although combination therapy of ICI and radiotherapy, chemotherapy can improve the efficacy of ICIs, the ORRs remain unsatisfactory. Thus, many efforts are underway to name reliable biomarkers to predict the response of ICIs. Nowadays, the best predictive biomarker identified is tumor mutational burden (TMB). TMB is highly relevant, as tumors with a higher mutational load exhibit higher levels of neoantigens capable of inducing anti-tumor immune responses (153). An elevated level of blood-based tumor mutational burden is positively correlated with the efficacy of Atezolizumab in treating NSCLC. Thus, it can be a clinically actionable biomarker for choosing appropriate patients (158). However, there are also some controversies about the predictive significance of TMB for ICIs. Since the inflamed tumors can undergo immune editing by promoting the loss of heterozygosity (LOH) in HLA locus (termed as HLA-LOH), whether high TMB tumors from highly inflamed cancers like NSCLC ultimately derive enhanced survival benefit ICIs. The latest results from 
CHECKMATE-227 and MYSTIC revealed that high TMB might not be a useful predictor for survival benefit from a combination of Nivolumab and Ipilimumab (11). Besides, the TIME with high $\mathrm{CD} 8^{+} \mathrm{T}$ cell infiltration (termed as "hot tumor") is associated with better ORR to ICIs (159). Similarly, the expression of intra-tumoral PDL1 is correlated with a better response to PD-1 or PDL1 antibodies in patients across multiple cancers (160). Moreover, in melanoma, baseline gut microbiota enriched with Faecalibacterium and other Firmicutes is associated with beneficial clinical response to Ipilimumab but more frequent occurrence of Ipilimumab-induced colitis (161).

\section{Prospect}

Significant advancements have been made in the field of cancer immunotherapy due to elegant research work conducted to elucidate the dynamics of TIME. ICIs and chimeric antigen receptor (CAR)- $\mathrm{T}$ cells are the most potential approaches to further improve the prognosis of cancer patients. However, despite the CAR-T cells achieved great success in treating hematological malignancies, they still face many obstacles in treating solid tumors. First, most CAR-T cells cannot recognize the tumor-specific antigens efficiently to exhibit adequate anti-tumor immunity. Furthermore, the physical barriers can prevent the afferent CAR-T cells to infiltrate into tumor microenvironment, and their poor infiltration cannot guarantee a valid killing efficiency. Last, those CAR-T cells successfully infiltrated into TIME cannot survive or proliferate as expected (162). ICIs exhibit anttumor effects by reactivating the immune cells in TIME, thus ICIs showed better clinical efficacies than CAR-T cells in treating solid tumors. Due to its promising efficacy and low toxicity, ICIs have opened a new era for cancer treatment. However, the dynamics and complexity of TIME still limit the clinical advancement of ICIs. Since some patients who lack TIL or IFN- $\gamma$ signaling in TIME are primarily resistant to ICIs, selecting the right patients is especially crucial for improving clinical efficacy. Thus, it is necessary to find new biomarkers for choosing proper patients to receive ICIs. Also, the immune-regulatory network can affect the efficacy of ICIs via inducing acquired resistance. Herein, establishing therapeutic strategies based on novel ICIs and other classical treatments is urgent to improve the efficacy of tumor immunotherapy further. Taken above, TIME plays a crucial role in regulating the efficacy and irAEs of ICIs.
Deeper comprehension is required for studying the TIME to improve the clinical application of ICIs.

\section{Acknowledgments}

Funding: This work was supported by the National Natural Science Foundations of China, Grant numbers: 81201607, 81871894.

\section{Footnote}

Conflicts of Interest: All authors have completed the ICMJE uniform disclosure form (available at http://dx.doi. org/10.21037/atm-20-3735). The authors have no conflicts of interest to declare.

Ethical Statement: The authors are accountable for all aspects of the work in ensuring that questions related to the accuracy or integrity of any part of the work are appropriately investigated and resolved.

Open Access Statement: This is an Open Access article distributed in accordance with the Creative Commons Attribution-NonCommercial-NoDerivs 4.0 International License (CC BY-NC-ND 4.0), which permits the noncommercial replication and distribution of the article with the strict proviso that no changes or edits are made and the original work is properly cited (including links to both the formal publication through the relevant DOI and the license). See: https://creativecommons.org/licenses/by-nc-nd/4.0/.

\section{References}

1. Siegel RL, Miller KD, Jemal A. Cancer statistics, 2020. CA Cancer J Clin 2020;70:7-30.

2. Hanahan D, Weinberg RA. Hallmarks of cancer: the next generation. Cell 2011;144:646-74.

3. Pedersen AW, Kopp KL, Andersen MH, et al. Immunoregulatory antigens-novel targets for cancer immunotherapy. Chin Clin Oncol 2018;7:19.

4. Coley WB. The Treatment of Inoperable Sarcoma by Bacterial Toxins (the Mixed Toxins of the Streptococcus erysipelas and the Bacillus prodigiosus). Proc R Soc Med 1910;3:1-48.

5. Re GL, Santeufemia DA, Re FL, et al. Interleukin-2 chronotherapy for metastatic renal cell carcinoma: Results of a phase I-II study. Cytokine 2020;128:154984.

6. Rosenberg SA. IL-2: the first effective immunotherapy for 
human cancer. J Immunol 2014;192:5451-8.

7. Rosenberg SA, Yang JC, Restifo NP. Cancer immunotherapy: moving beyond current vaccines. Nat Med 2004;10:909-15.

8. O'Donnell JS, Teng MWL, Smyth MJ. Cancer immunoediting and resistance to $\mathrm{T}$ cell-based immunotherapy. Nat Rev Clin Oncol 2019;16:151-67.

9. Fong L, Small EJ. Anti-cytotoxic T-lymphocyte antigen- 4 antibody: the first in an emerging class of immunomodulatory antibodies for cancer treatment. J Clin Oncol 2008;26:5275-83.

10. Wang S, Chen L. Immunobiology of cancer therapies targeting CD137 and B7-H1/PD-1 cosignal pathways. Curr Top Microbiol Immunol 2011;344:245-67.

11. Hegde PS, Chen DS. Top 10 Challenges in Cancer Immunotherapy. Immunity 2020;52:17-35.

12. Thorsson V, Gibbs DL, Brown SD, et al. The Immune Landscape of Cancer. Immunity 2018;48:812-830.e14.

13. Locy H, de Mey S, de Mey W, et al. Immunomodulation of the Tumor Microenvironment: Turn Foe Into Friend. Front Immunol 2018;9:2909.

14. Eckstein M, Gupta S. New insights in predictive determinants of the tumor immune microenvironment for immune checkpoint inhibition: a never ending story? Ann Transl Med 2019;7:S135.

15. Chen L. Co-inhibitory molecules of the B7-CD28 family in the control of T-cell immunity. Nat Rev Immunol 2004;4:336-47.

16. Zou W, Chen L. Inhibitory B7-family molecules in the tumour microenvironment. Nat Rev Immunol 2008;8:467-77.

17. Keir ME, Butte MJ, Freeman GJ, et al. PD-1 and its ligands in tolerance and immunity. Annu Rev Immunol 2008;26:677-704.

18. Iwai $Y$, Ishida $M$, Tanaka $Y$, et al. Involvement of PD$\mathrm{L} 1$ on tumor cells in the escape from host immune system and tumor immunotherapy by PD-L1 blockade. Proc Natl Acad Sci U S A 2002;99:12293-7.

19. Sica GL, Choi IH, Zhu G, et al. B7-H4, a molecule of the B7 family, negatively regulates $T$ cell immunity. Immunity 2003;18:849-61.

20. Li J, Lee Y, Li Y, et al. Co-inhibitory Molecule B7 Superfamily Member 1 Expressed by Tumor-Infiltrating Myeloid Cells Induces Dysfunction of Anti-tumor CD8(+) T Cells. Immunity 2018;48:773-786.e5.

21. Flies DB, Wang S, Xu H, et al. Cutting edge: A monoclonal antibody specific for the programmed death-1 homolog prevents graft-versus-host disease in mouse models. J Immunol 2011;187:1537-41.

22. Johnston RJ, Su LJ, Pinckney J, et al. VISTA is an acidic pH-selective ligand for PSGL-1. Nature 2019;574:565-70.

23. Wang J, Wu G, Manick B, et al. VSIG-3 as a ligand of VISTA inhibits human T-cell function. Immunology 2019;156:74-85.

24. Goldberg MV, Drake CG. LAG-3 in Cancer Immunotherapy. Curr Top Microbiol Immunol 2011;344:269-78.

25. Nguyen LT, Ohashi PS. Clinical blockade of PD1 and LAG3--potential mechanisms of action. Nat Rev Immunol 2015;15:45-56.

26. Das M, Zhu C, Kuchroo VK. Tim-3 and its role in regulating anti-tumor immunity. Immunol Rev 2017;276:97-111.

27. Shevyrev D, Tereshchenko V. Treg Heterogeneity, Function, and Homeostasis. Front Immunol 2020;10:3100.

28. Wang W, Lau R, Yu D, et al. PD1 blockade reverses the suppression of melanoma antigen-specific CTL by CD4+ CD25(Hi) regulatory T cells. Int Immunol 2009;21:1065-77.

29. Takeuchi $Y$, Nishikawa $H$. Roles of regulatory $T$ cells in cancer immunity. Int Immunol 2016;28:401-9.

30. Wang Y, Wang H, Yao H, et al. Regulation of PD-L1: Emerging Routes for Targeting Tumor Immune Evasion. Front Pharmacol 2018;9:536.

31. Budczies J, Bockmayr M, Denkert C, et al. Pan-cancer analysis of copy number changes in programmed death-ligand 1 (PD-L1, CD274) - associations with gene expression, mutational load, and survival. Genes Chromosomes Cancer 2016;55:626-39.

32. Wu Y, Zhao T, Jia Z, et al. Polymorphism of the programmed death-ligand 1 gene is associated with its protein expression and prognosis in gastric cancer. $\mathrm{J}$ Gastroenterol Hepatol 2019;34:1201-7.

33. Micevic G, Thakral D, McGeary M, et al. PD-L1 methylation regulates PD-L1 expression and is associated with melanoma survival. Pigment Cell Melanoma Res 2019;32:435-40.

34. Patel JJ, Levy DA, Nguyen SA, et al. Impact of PD-L1 expression and human papillomavirus status in anti-PD1/ PDL1 immunotherapy for head and neck squamous cell carcinoma-Systematic review and meta-analysis. Head Neck 2020;42:774-86.

35. Wrangle J, Wang W, Koch A, et al. Alterations of immune response of Non-Small Cell Lung Cancer with Azacytidine. Oncotarget 2013;4:2067-79.

36. Wang YF, Liu F, Sherwin S, et al. Cooperativity of 
HOXA5 and STAT3 Is Critical for HDAC8 InhibitionMediated Transcriptional Activation of PD-L1 in Human Melanoma Cells. J Invest Dermatol 2018;138:922-32.

37. Lu C, Paschall AV, Shi H, et al. The MLL1-H3K4me3 Axis-Mediated PD-L1 Expression and Pancreatic Cancer Immune Evasion. J Natl Cancer Inst 2017;109:djw283.

38. Vasudevan S, Tong Y, Steitz JA. Switching from repression to activation: microRNAs can up-regulate translation. Science 2007;318:1931-4.

39. Ashizawa M, Okayama H, Ishigame T, et al. miRNA148a-3p Regulates Immunosuppression in DNA Mismatch Repair-Deficient Colorectal Cancer by Targeting PD-L1. Mol Cancer Res 2019;17:1403-13.

40. Mastroianni J, Stickel N, Andrlova H, et al. miR146a Controls Immune Response in the Melanoma Microenvironment. Cancer Res 2019;79:183-95.

41. Gao L, Guo Q, Li X, et al. MiR-873/PD-L1 axis regulates the stemness of breast cancer cells. EBioMedicine 2019;41:395-407.

42. Xu J, Meng Q, Li X, et al. Long Noncoding RNA MIR17HG Promotes Colorectal Cancer Progression via miR-17-5p. Cancer Res 2019;79:4882-95.

43. Zhou WY, Zhang MM, Liu C, et al. Long noncoding RNA LINC00473 drives the progression of pancreatic cancer via upregulating programmed death-ligand 1 by sponging microRNA-195-5p. J Cell Physiol 2019;234:23176-89.

44. Takigawa N, Ochi N, Yamane H. Histology versus cytology: PD-L1 testing in non-small cell lung cancer. Transl Lung Cancer Res 2018;7:S225-7.

45. Peng S, Wang R, Zhang X, et al. EGFR-TKI resistance promotes immune escape in lung cancer via increased PDL1 expression. Mol Cancer 2019;18:165.

46. Wang J, Jia Y, Zhao S, et al. BIN1 reverses PD-L1mediated immune escape by inactivating the c-MYC and EGFR/MAPK signaling pathways in non-small cell lung cancer. Oncogene 2017;36:6235-43.

47. Shen X, Zhang L, Li J, et al. Recent Findings in the Regulation of Programmed Death Ligand 1 Expression. Front Immunol 2019;10:1337.

48. Platanias LC. Mechanisms of type-I- and type-IIinterferon-mediated signalling. Nat Rev Immunol 2005;5:375-86.

49. Chiossone L, Dumas PY, Vienne M, et al. Natural killer cells and other innate lymphoid cells in cancer. Nat Rev Immunol 2018;18:671-88.

50. Vivier E, Raulet DH, Moretta A, et al. Innate or adaptive immunity? The example of natural killer cells. Science
2011;331:44-9.

51. Grossenbacher SK, Canter RJ, Murphy WJ. Natural killer cell immunotherapy to target stem-like tumor cells. J Immunother Cancer 2016;4:19.

52. $\mathrm{Hu} W$, Wang G, Huang D, et al. Cancer Immunotherapy Based on Natural Killer Cells: Current Progress and New Opportunities. Front Immunol 2019;10:1205.

53. Olkhanud PB, Baatar D, Bodogai M, et al. Breast cancer lung metastasis requires expression of chemokine receptor CCR4 and regulatory T cells. Cancer Res 2009;69:5996-6004.

54. Sanchez-Correa B, Lopez-Sejas N, Duran E, et al. Modulation of NK cells with checkpoint inhibitors in the context of cancer immunotherapy. Cancer Immunol Immunother 2019;68:861-70.

55. Sheu BC, Chiou SH, Lin HH, et al. Up-regulation of inhibitory natural killer receptors CD94/NKG2A with suppressed intracellular perforin expression of tumorinfiltrating CD8+ T lymphocytes in human cervical carcinoma. Cancer Res 2005;65:2921-9.

56. Rapaport AS, Schriewer J, Gilfillan S, et al. The Inhibitory Receptor NKG2A Sustains Virus-Specific CD8(+) T Cells in Response to a Lethal Poxvirus Infection. Immunity 2015;43:1112-24.

57. André P, Denis C, Soulas C, et al. Anti-NKG2A mAb Is a Checkpoint Inhibitor that Promotes Anti-tumor Immunity by Unleashing Both T and NK Cells. Cell 2018;175:17311743.e13.

58. Sharma P, Hu-Lieskovan S, Wargo JA, et al. Primary, Adaptive, and Acquired Resistance to Cancer Immunotherapy. Cell 2017;168:707-23.

59. Noman MZ, Desantis G, Janji B, et al. PD-L1 is a novel direct target of HIF-1alpha, and its blockade under hypoxia enhanced MDSC-mediated T cell activation. J Exp Med 2014;211:781-90.

60. Parker KH, Beury DW, Ostrand-Rosenberg S. MyeloidDerived Suppressor Cells: Critical Cells Driving Immune Suppression in the Tumor Microenvironment. Adv Cancer Res 2015;128:95-139.

61. Weber R, Fleming V, Hu X, et al. Myeloid-Derived Suppressor Cells Hinder the Anti-Cancer Activity of Immune Checkpoint Inhibitors. Front Immunol 2018;9:1310.

62. Le Mercier I, Chen W, Lines JL, et al. VISTA Regulates the Development of Protective Antitumor Immunity. Cancer Res 2014;74:1933-44.

63. Noy R, Pollard JW. Tumor-associated macrophages: from mechanisms to therapy. Immunity 2014;41:49-61. 
64. Chanmee T, Ontong P, Konno K, et al. Tumorassociated macrophages as major players in the tumor microenvironment. Cancers (Basel) 2014;6:1670-90.

65. Jeannin P, Paolini L, Adam C, et al. The roles of CSFs on the functional polarization of tumor-associated macrophages. Febs j 2018;285:680-99.

66. Kryczek I, Zou L, Rodriguez P, et al. B7-H4 expression identifies a novel suppressive macrophage population in human ovarian carcinoma. J Exp Med 2006;203:871-81.

67. Sica A, Schioppa T, Mantovani A, et al. Tumour-associated macrophages are a distinct M2 polarised population promoting tumour progression: potential targets of anticancer therapy. Eur J Cancer 2006;42:717-27.

68. Zong Z, Zou J, Mao R, et al. M1 Macrophages Induce PD-L1 Expression in Hepatocellular Carcinoma Cells Through IL-1beta Signaling. Front Immunol 2019;10:1643.

69. Zhu Y, Knolhoff BL, Meyer MA, et al. CSF1/CSF1R blockade reprograms tumor-infiltrating macrophages and improves response to $\mathrm{T}$-cell checkpoint immunotherapy in pancreatic cancer models. Cancer Res 2014;74:5057-69.

70. Chen Y, Song Y, Du W, et al. Tumor-associated macrophages: an accomplice in solid tumor progression. J Biomed Sci 2019;26:78.

71. Li X, Liu R, Su X, et al. Harnessing tumor-associated macrophages as aids for cancer immunotherapy. Mol Cancer 2019;18:177.

72. Geiger R, Rieckmann JC, Wolf T, et al. L-Arginine Modulates T Cell Metabolism and Enhances Survival and Anti-tumor Activity. Cell 2016;167:829-842.e13.

73. De Palma M, Lewis CE. Macrophage regulation of tumor responses to anticancer therapies. Cancer Cell 2013;23:277-86.

74. DeNardo DG, Ruffell B. Macrophages as regulators of tumour immunity and immunotherapy. Nat Rev Immunol 2019;19:369-82.

75. Fridlender ZG, Sun J, Kim S, et al. Polarization of tumorassociated neutrophil phenotype by TGF-beta: "N1" versus "N2" TAN. Cancer Cell 2009;16:183-94.

76. Gershkovitz M, Fainsod-Levi T, Zelter T, et al. TRPM2 modulates neutrophil attraction to murine tumor cells by regulating CXCL2 expression. Cancer Immunol Immunother 2019;68:33-43.

77. Sun B, Qin W, Song M, et al. Neutrophil Suppresses Tumor Cell Proliferation via Fas /Fas Ligand Pathway Mediated Cell Cycle Arrested. Int J Biol Sci 2018;14:2103-13.

78. Mishalian I, Bayuh R, Levy L, et al. Tumor-associated neutrophils (TAN) develop pro-tumorigenic properties during tumor progression. Cancer Immunol Immunother 2013;62:1745-56.

79. Casbon AJ, Reynaud D, Park C, et al. Invasive breast cancer reprograms early myeloid differentiation in the bone marrow to generate immunosuppressive neutrophils. Proc Natl Acad Sci U S A 2015;112:E566-75.

80. Coffelt SB, Wellenstein MD, de Visser KE. Neutrophils in cancer: neutral no more. Nat Rev Cancer 2016;16:431-46.

81. Tsuda Y, Fukui H, Asai A, et al. An immunosuppressive subtype of neutrophils identified in patients with hepatocellular carcinoma. J Clin Biochem Nutr 2012;51:204-12.

82. Dumitru CA, Fechner MK, Hoffmann TK, et al. A novel p38-MAPK signaling axis modulates neutrophil biology in head and neck cancer. J Leukoc Biol 2012;91:591-8.

83. Jia Y, Wang H, Wang Y, et al. Low expression of Bin1, along with high expression of IDO in tumor tissue and draining lymph nodes, are predictors of poor prognosis for esophageal squamous cell cancer patients. Int J Cancer 2015;137:1095-106.

84. Komiya T, Huang CH. Updates in the Clinical Development of Epacadostat and Other Indoleamine 2,3-Dioxygenase 1 Inhibitors (IDO1) for Human Cancers. Front Oncol 2018;8:423.

85. Fukumura D, Kloepper J, Amoozgar Z, et al. Enhancing cancer immunotherapy using antiangiogenics: opportunities and challenges. Nat Rev Clin Oncol 2018;15:325-40.

86. Campesato LF, Merghoub T. Antiangiogenic therapy and immune checkpoint blockade go hand in hand. Ann Transl Med 2017;5:497.

87. Costa A, Kieffer Y, Scholer-Dahirel A, et al. Fibroblast heterogeneity and immunosuppressive environment in human breast cancer. Cancer Cell 2018;33:463-479.e10.

88. Tsuyada A, Chow A, Wu J, et al. CCL2 mediates cross-talk between cancer cells and stromal fibroblasts that regulates breast cancer stem cells. Cancer Res 2012;72:2768-79.

89. De Jaeghere EA, Denys HG, De Wever O. Fibroblasts Fuel Immune Escape in the Tumor Microenvironment. Trends Cancer 2019;5:704-23.

90. Xia L, Liu Y, Wang Y. PD-1/PD-L1 Blockade Therapy in Advanced Non-Small-Cell Lung Cancer: Current Status and Future Directions. Oncologist 2019;24:S31-S41.

91. Robert C, Thomas L, Bondarenko I, et al. Ipilimumab plus dacarbazine for previously untreated metastatic melanoma. N Engl J Med 2011;364:2517-26.

92. Topalian SL, Hodi FS, Brahmer JR, et al. Safety, activity, 
and immune correlates of anti-PD-1 antibody in cancer. $\mathrm{N}$ Engl J Med 2012;366:2443-54.

93. Topalian SL, Sznol M, McDermott DF, et al. Survival, durable tumor remission, and long-term safety in patients with advanced melanoma receiving nivolumab. J Clin Oncol 2014;32:1020-30.

94. Sul J, Blumenthal GM, Jiang X, et al. FDA Approval Summary: Pembrolizumab for the Treatment of Patients With Metastatic Non-Small Cell Lung Cancer Whose Tumors Express Programmed Death-Ligand 1. Oncologist 2016;21:643-50.

95. Mok TSK, Wu YL, Kudaba I, et al. Pembrolizumab versus chemotherapy for previously untreated, PD-L1expressing, locally advanced or metastatic non-small-cell lung cancer (KEYNOTE-042): a randomised, open-label, controlled, phase 3 trial. Lancet 2019;393:1819-30.

96. Carbone DP, Reck M, Paz-Ares L, et al. First-line nivolumab in stage IV or recurrent non-small-cell lung cancer. N Engl J Med 2017;376:2415-26.

97. Brahmer J, Reckamp KL, Baas P, et al. Nivolumab versus Docetaxel in advanced squamous-cell non-small-cell lung cancer. N Engl J Med 2015;373:123-35.

98. Borghaei H, Paz-Ares L, Horn L, et al. Nivolumab versus Docetaxel in advanced nonsquamous non-small-cell lung cancer. N Engl J Med 2015;373:1627-39.

99. Socinski MA, Jotte RM, Cappuzzo F, et al. Atezolizumab for first-line treatment of metastatic nonsquamous NSCLC. N Engl J Med 2018;378:2288-301.

100. Rittmeyer A, Barlesi F, Waterkamp D, et al. Atezolizumab versus docetaxel in patients with previously treated non-small-cell lung cancer (OAK): a phase 3, openlabel, multicentre randomised controlled trial. Lancet 2017;389:255-65.

101. Robert C, Long GV, Brady B, et al. Nivolumab in previously untreated melanoma without BRAF mutation. N Engl J Med 2015;372:320-30.

102.Hamid O, Robert C, Daud A, et al. Safety and tumor responses with lambrolizumab (anti-PD-1) in melanoma. N Engl J Med 2013;369:134-44.

103. Eggermont AMM, Blank CU, Mandala M, et al. Adjuvant Pembrolizumab versus Placebo in Resected Stage III Melanoma. N Engl J Med 2018;378:1789-801.

104.Eggermont AM, Chiarion-Sileni V, Grob JJ, et al. Adjuvant ipilimumab versus placebo after complete resection of high-risk stage III melanoma (EORTC 18071): a randomised, double-blind, phase 3 trial. Lancet Oncol 2015;16:522-30.

105. Motzer RJ, Escudier B, McDermott DF, et al. Nivolumab versus Everolimus in Advanced Renal-Cell Carcinoma. N Engl J Med 2015;373:1803-13.

106. Nanda R, Chow LQ, Dees EC, et al. Pembrolizumab in Patients With Advanced Triple-Negative Breast Cancer: Phase Ib KEYNOTE-012 Study. J Clin Oncol 2016;34:2460-7.

107. Adams S, Loi S, Toppmeyer D, et al. Pembrolizumab monotherapy for previously untreated, PD-L1-positive, metastatic triple-negative breast cancer: cohort B of the phase II KEYNOTE-086 study. Ann Oncol 2019;30:405-11.

108. Inman BA, Longo TA, Ramalingam S, et al. Atezolizumab: A PD-L1-Blocking Antibody for Bladder Cancer. Clin Cancer Res 2017;23:1886-90.

109.Jaffray DA. Image-guided radiotherapy: from current concept to future perspectives. Nat Rev Clin Oncol 2012;9:688-99.

110. Golden EB, Apetoh L. Radiotherapy and immunogenic cell death. Semin Radiat Oncol 2015;25:11-7.

111. Reits EA, Hodge JW, Herberts CA, et al. Radiation modulates the peptide repertoire, enhances $\mathrm{MHC}$ class I expression, and induces successful antitumor immunotherapy. J Exp Med 2006;203:1259-71.

112. Filatenkov A, Baker J, Mueller AM, et al. Ablative Tumor Radiation Can Change the Tumor Immune Cell Microenvironment to Induce Durable Complete Remissions. Clin Cancer Res 2015;21:3727-39.

113. Oweida A, Hararah MK, Phan A, et al. Resistance to Radiotherapy and PD-L1 Blockade Is Mediated by TIM3 Upregulation and Regulatory T-Cell Infiltration. Clin Cancer Res 2018;24:5368-80.

114. Shaverdian N, Lisberg AE, Bornazyan K, et al. Previous radiotherapy and the clinical activity and toxicity of pembrolizumab in the treatment of non-small-cell lung cancer: a secondary analysis of the KEYNOTE-001 phase 1 trial. Lancet Oncol 2017;18:895-903.

115. Formenti SC, Rudqvist NP, Golden E, et al. Radiotherapy induces responses of lung cancer to CTLA-4 blockade. Nat Med 2018;24:1845-51.

116. Kanda S, Goto K, Shiraishi H, et al. Safety and efficacy of nivolumab and standard chemotherapy drug combination in patients with advanced non-small-cell lung cancer: a four arms phase Ib study. Ann Oncol 2016;27:2242-50.

117. Tran L, Allen CT, Xiao R, et al. Cisplatin Alters Antitumor Immunity and Synergizes with PD-1/PD-L1 Inhibition in Head and Neck Squamous Cell Carcinoma. Cancer Immunol Res 2017;5:1141-51.

118. Langer CJ, Gadgeel SM, Borghaei H, et al. Carboplatin and pemetrexed with or without pembrolizumab for 
advanced, non-squamous non-small-cell lung cancer: a randomised, phase 2 cohort of the open-label KEYNOTE-021 study. Lancet Oncol 2016;17:1497-508.

119.Reck M. Pembrolizumab as first-line therapy for metastatic non-small-cell lung cancer. Immunotherapy 2018;10:93-105.

120.Horn L, Mansfield AS, Szczesna A, et al. First-Line Atezolizumab plus Chemotherapy in Extensive-Stage Small-Cell Lung Cancer. N Engl J Med 2018;379:2220-9.

121.Paz-Ares L, Dvorkin M, Chen Y, et al. Durvalumab plus platinum-etoposide versus platinum-etoposide in firstline treatment of extensive-stage small-cell lung cancer (CASPIAN): a randomised, controlled, open-label, phase 3 trial. Lancet 2019;394:1929-39.

122. Schmid P, Adams S, Rugo HS, et al. Atezolizumab and Nab-Paclitaxel in Advanced Triple-Negative Breast Cancer. N Engl J Med 2018;379:2108-21.

123. Lee M, Ryoo B, Hsu C, et al. Randomised efficacy and safety results from atezolizumab (Atezo) + bevacizumab (Bev) in patients (pts) with previously untreated, hepatocellular carcinoma (HCC). ESMO 2019 Congress. Ann Oncol 2019;30:v851-v934.

124. Motzer RJ, Penkov K, Haanen JBAG, et al. LBA6_ PRJAVELIN renal 101: A randomized phase III study of avelumab + axitinib vs sunitinib as first-line treatment of advanced renal cell carcionoma (aRCC). Ann Oncol 2018;29:mdy424.036.

125.Kim TK, Herbst RS, Chen L. Defining and Understanding Adaptive Resistance in Cancer Immunotherapy. Trends Immunol 2018;39:624-31.

126.Lai Q, Wang H, Li A, et al. Decitibine improve the efficiency of anti-PD-1 therapy via activating the response to IFN/PD-L1 signal of lung cancer cells. Oncogene 2018;37:2302-12.

127.Pan Y, Fei Q, Xiong P, et al. Synergistic inhibition of pancreatic cancer with anti-PD-L1 and c-Myc inhibitor JQ1. Oncoimmunology 2019;8:e1581529.

128. Schumacher TN, Schreiber RD. Neoantigens in cancer immunotherapy. Science 2015;348:69-74.

129.Spranger S, Bao R, Gajewski TF. Melanoma-intrinsic beta-catenin signalling prevents anti-tumour immunity. Nature 2015;523:231-5.

130. Gao J, Shi LZ, Zhao H, et al. Loss of IFN-gamma pathway genes in tumor cells as a mechanism of resistance to Anti-CTLA-4 therapy. Cell 2016;167:397-404.e9.

131.Liu C, Peng W, Xu C, et al. BRAF inhibition increases tumor infiltration by $\mathrm{T}$ cells and enhances the antitumor activity of adoptive immunotherapy in mice. Clin Cancer
Res 2013;19:393-403.

132. Popat S, Grohe C, Corral J, et al. Anti-angiogenic agents in the age of resistance to immune checkpoint inhibitors: Do they have a role in non-oncogene-addicted non-small cell lung cancer? Lung Cancer 2020;144:76-84.

133. Khair DO, Bax HJ, Mele S, et al. Combining immune checkpoint inhibitors: established and emerging targets and strategies to improve outcomes in melanoma. Front Immunol 2019;10:453.

134.Zaretsky JM, Garcia-Diaz A, Shin DS, et al. Mutations associated with acquired resistance to PD-1 blockade in melanoma. N Engl J Med 2016;375:819-29.

135. Gao J, Ward JF, Pettaway CA, et al. VISTA is an inhibitory immune checkpoint that is increased after ipilimumab therapy in patients with prostate cancer. Nat Med 2017;23:551-5.

136.Zhang Y, Xiang C, Wang Y, et al. PD-L1 promoter methylation mediates the resistance response to anti-PD-1 therapy in NSCLC patients with EGFR-TKI resistance. Oncotarget 2017;8:101535-44.

137.Jhaveri KD, Wanchoo R. Adverse events associated with immune checkpoint inhibitors. JAMA 2019;321:1218-9.

138. Wang DY, Salem JE, Cohen JV, et al. Fatal toxic effects associated with immune checkpoint inhibitors: a systematic review and meta-analysis. JAMA Oncol 2018;4:1721-8.

139. Das S, Johnson DB. Immune-related adverse events and anti-tumor efficacy of immune checkpoint inhibitors. J Immunother Cancer 2019;7:306.

140.Johnson DB, Balko JM. Biomarkers for immunotherapy toxicity: are cytokines the answer? Clin Cancer Res 2019;25:1452-4.

141. Wolchok JD, Chiarion-Sileni V, Gonzalez R, et al. Overall Survival with Combined Nivolumab and Ipilimumab in Advanced Melanoma. N Engl J Med 2017;377:1345-56.

142. Grangeon M, Tomasini P, Chaleat S, et al. Association between immune-related adverse events and efficacy of immune checkpoint inhibitors in non-small-cell lung cancer. Clin Lung Cancer 2019;20:201-7.

143. Borcoman E, Nandikolla A, Long G, et al. Patterns of Response and Progression to Immunotherapy. Am Soc Clin Oncol Educ Book 2018;38:169-78.

144. Maher VE, Fernandes LL, Weinstock C, et al. Analysis of the Association Between Adverse Events and Outcome in Patients Receiving a Programmed Death Protein 1 or Programmed Death Ligand 1 Antibody. J Clin Oncol 2019;37:2730-7.

145. Berner F, Bomze D, Diem S, et al. Association of checkpoint inhibitor-induced toxic effects with shared 
cancer and tissue antigens in non-small cell lung cancer. JAMA Oncol 2019;5:1043-7.

146. Faje AT, Lawrence D, Flaherty K, et al. High-dose glucocorticoids for the treatment of ipilimumab-induced hypophysitis is associated with reduced survival in patients with melanoma. Cancer 2018;124:3706-14.

147.Hodi FS, Lee S, McDermott DF, et al. Ipilimumab plus sargramostim vs ipilimumab alone for treatment of metastatic melanoma: a randomized clinical trial. Jama 2014;312:1744-53.

148. Santini FC, Rizvi H, Plodkowski AJ, et al. Safety and Efficacy of Re-treating with Immunotherapy after Immune-Related Adverse Events in Patients with NSCLC. Cancer Immunol Res 2018;6:1093-9.

149. Andrews LP, Marciscano AE, Drake CG, et al. LAG3 (CD223) as a cancer immunotherapy target. Immunol Rev 2017;276:80-96.

150.van Hall T, Andre P, Horowitz A, et al. Monalizumab: inhibiting the novel immune checkpoint NKG2A. J Immunother Cancer 2019;7:263.

151. Terszowski G, Klein C, Schmied L, et al. How to outsmart NK cell tolerance. Oncoimmunology 2015;4:e1016708.

152.Lee HT, Lee SH, Heo YS. Molecular Interactions of Antibody Drugs Targeting PD-1, PD-L1, and CTLA-4 in Immuno-Oncology. Molecules 2019;24:1190.

153.Hellmann MD, Paz-Ares L, Bernabe Caro R, et al. Nivolumab plus Ipilimumab in Advanced Non-Small-Cell Lung Cancer. N Engl J Med 2019;381:2020-31.

154.Larkin J, Chiarion-Sileni V, Gonzalez R, et al. Five-Year Survival with Combined Nivolumab and Ipilimumab in Advanced Melanoma. N Engl J Med

Cite this article as: Jia Y, Liu L, Shan B. Future of immune checkpoint inhibitors: focus on tumor immune microenvironment. Ann Transl Med 2020;8(17):1095. doi: 10.21037/atm-20-3735
2019;381:1535-46.

155.Janjigian YY, Bendell J, Calvo E, et al. CheckMate-032 Study: Efficacy and Safety of Nivolumab and Nivolumab Plus Ipilimumab in Patients With Metastatic Esophagogastric Cancer. J Clin Oncol 2018;36:2836-44.

156.Li H, Bullock K, Gurjao C, et al. Metabolomic adaptations and correlates of survival to immune checkpoint blockade. Nat Commun 2019;10:4346.

157.Long GV, Dummer R, Hamid O, et al. Epacadostat plus pembrolizumab versus placebo plus pembrolizumab in patients with unresectable or metastatic melanoma (ECHO-301/KEYNOTE-252): a phase 3, randomised, double-blind study. Lancet Oncol 2019;20:1083-97.

158. Gandara DR, Paul SM, Kowanetz M, et al. Bloodbased tumor mutational burden as a predictor of clinical benefit in non-small-cell lung cancer patients treated with atezolizumab. Nat Med 2018;24:1441-8.

159. Tumeh PC, Harview CL, Yearley JH, et al. PD-1 blockade induces responses by inhibiting adaptive immune resistance. Nature 2014;515:568-71.

160. Gandini S, Massi D, Mandala M. PD-L1 expression in cancer patients receiving anti PD-1/PD-L1 antibodies: A systematic review and meta-analysis. Crit Rev Oncol Hematol 2016;100:88-98.

161. Chaput N, Lepage P, Coutzac C, et al. Baseline gut microbiota predicts clinical response and colitis in metastatic melanoma patients treated with ipilimumab. Ann Oncol 2017;28:1368-79.

162. Martinez M, Moon EK. CAR T cells for solid tumors: new strategies for finding, infiltrating, and surviving in the tumor microenvironment. Front Immunol 2019;10:128. 